\title{
miR-124 inhibits cell proliferation, migration and invasion by directly targeting SOX9 in lung adenocarcinoma
}

\author{
XIAOYING WANG ${ }^{1}$, YANLI LIU ${ }^{2}$, XIAOLI LIU ${ }^{3}$, JINGYAN YANG ${ }^{1}$, GUOXIN TENG $^{1}$, \\ LULU ZHANG ${ }^{1}$ and CHENGJUN ZHOU ${ }^{1}$
}

\author{
${ }^{1}$ Department of Pathology, The Second Hospital of Shandong University, Jinan, Shandong 250033; \\ ${ }^{2}$ Provincial Key Laboratory of Radio-Oncology, Shandong Cancer Hospital and Institute, Jinan, Shandong 250117; \\ ${ }^{3}$ Department of Clinical Laboratory, The Second Hospital of Shandong University, Jinan, Shandong 250033, P.R. China
}

Received November 28, 2015; Accepted January 5, 2016

DOI: $10.3892 / o r .2016 .4648$

\begin{abstract}
Accumulating evidence indicates that dysregulation of microRNAs (miRNAs) may contribute to the initiation and progression of cancer. However, the role of miR-124 in lung adenocarcinoma (ADC) and the underlying mechanisms through which miR-124 exerts its functions are not completely understood. In the present study, we detected miR-124 and SOX9 expression in lung ADC tissues. The results showed that miR-124 was significantly downregulated in the lung ADC tissues compared with that noted in the corresponding non-cancerous lung tissues and the level of SOX9 protein was inversely associated with the expression of miR-124. The study in human lung ADC cell line A549 demonstrated that upregulation of miR-124 could inhibit cell proliferation, migration and invasion. The bioinformatic analysis showed that there was a putative miR-124 binding site in the $3^{\prime}$ untranslated region (3'UTR) of SOX9. Using a luciferase reporter assay, we verified that SOX9 is a direct target of miR-124. Furthermore, overexpression of miR-124 repressed SOX9 expression, whereas inhibition of miR-124 increased expression of SOX9 in the A549 cells. Finally, we identified that SOX9 was a functional mediator of miR-124 in A549 cells. Taken together, our results suggest that miR-124 functions as a tumor suppressor in lung ADC by directly targeting SOX9 and it may be a promising candidate for miR-based therapy against lung ADC.
\end{abstract}

\section{Introduction}

Lung cancer is the leading cause of cancer-related death worldwide. Despite advances in surgical techniques and strategies of chemoradiotherapy and targeted therapy, the 5-year survival rate of patients with lung cancer remains low (less than 15\%).

Correspondence to: Professor Chengjun Zhou, Department of Pathology, The Second Hospital of Shandong University, 247 Beiyuan Street, Jinan, Shandong 250033, P.R. China

E-mail:wang_xy1896@163.com

Key words: miR-124, SOX9, lung adenocarcinoma, proliferation, migration, invasion
Therefore, it is important to investigate the molecular mechanisms underlying lung cancer that would lead to new therapies for improving patient survival and quality of life.

MicroRNAs (miRNAs) are a class of small endogenous non-coding RNAs that regulate the expression of their target genes at the post-transcriptional level. miRNAs play important roles in various biological processes such as cell proliferation, migration, development, differentiation and apoptosis $(1,2)$. Beyond the involvement in physiological processes, accumulating studies also strongly suggest that the dysregulation of miRNAs may contribute to the initiation and progression of cancer (3). miR-124 is the most abundant miRNA expressed in neuronal cells and plays a role in neuronal differentiation. In addition, emerging evidence implicates miR-124 in the pathogenesis of several human malignancies. It has been reported that miR-124 can act as a putative tumor suppressor in prostate (4), breast (5), colorectal (6), cervical (7) and gastric cancer (8), nasopharyngeal carcinoma (9), bladder (10) and ovarian cancer (11) and hepatocellular carcinoma (12). Several studies have also shown that miR-124 is downregulated in lung cancer (13-15). However, the role of miR-124 in lung adenocarcinoma (ADC) and the underlying mechanisms through which miR-124 exerts its functions remain unclear.

SOX9, which is one of the members of the SRY boxcontaining (SOX) family, plays a key role in developmental processes, including chondrogenesis, neurogenesis and male sex determination $(16,17)$. Subsequently, increasing evidence has revealed that $\mathrm{SOX} 9$ is involved in the development of cancer. It has been reported that SOX9 is upregulated in colorectal cancer (18), lung ADC (19), prostate (20) and breast cancer (21), and pancreatic ductal ADC (22), whereas it is downregulated in cervical carcinoma (23). Therefore, SOX9 may function as an oncogene or tumor suppressor depending on tumor origin. Intriguingly, several miRs, including miR-145 (24), miR-32 (25) and miR-101 (26), have been found to target SOX9 in different tissues. Real et al (27) showed that miR-124 can regulate the expression of SOX9 in developing mouse ovarian cells. However, the potential regulatory effect of miR-124 on SOX9 expression in lung ADC has not been verified.

In the present study, our results showed that miR-124 was substantially decreased in lung ADC tissues, and upregulation of miR-124 inhibited the proliferation, migration and invasion 
of A549 cells. Moreover, our data demonstrated that SOX9 is a direct target gene and functional mediator of miR-124 in lung $\mathrm{ADC}$ cells.

\section{Materials and methods}

Patients and tissue specimens. Sixty-five paired ADC lung samples and adjacent non-tumorous lung tissues were obtained from patients undergoing surgical resection and histologically confirmed by two pathologists at the Second Hospital of Shandong University. These tissue samples were immediately frozen in liquid nitrogen and then stored at $-80^{\circ} \mathrm{C}$ until protein and RNA extraction. Written informed consent was obtained from all patients, and the present study was approved by the Institutional Research Ethics Committee of the Second Hospital of Shandong University.

Cell culture and DNA construction. The human lung ADC cell line A549 was purchased from the Cell Bank of the Chinese Academy of Medical Sciences (Beijing, China), and was routinely cultured in Dulbecco's modified Eagle's medium (DMEM) supplemented with $10 \%$ heat-inactivated fetal bovine serum (FBS) (both from Invitrogen, Carlsbad, CA, USA), $100 \mathrm{U} / \mathrm{ml}$ penicillin and $100 \mu \mathrm{g} / \mathrm{ml}$ streptomycin (both from Sigma-Aldrich, St. Louis, MO, USA). The cells were incubated at $37^{\circ} \mathrm{C}$ in a humidified atmosphere with $5 \% \mathrm{CO}_{2}$. The SOX9 plasmid was constructed according to a previous study (19). Briefly, the coding sequences of SOX9 were PCR amplified with the forward primer, 5'-GGATCCCATGAATC TCCTGGACCCCT-3' and the reverse primer, 5'-GAATTC TCAAGGTCGAGTGAGCTGTGTGT-3'; and then subcloned into the pCMV-Tag2 expression vector (Stratagene, La Jolla, CA, USA).

Real-time RT-PCR. To determine the relative expression level of miR-124, total RNA was extracted from the tissues and cultured cells using the mirVana ${ }^{\mathrm{TM}}$ miRNA isolation kit (AM1560; Ambion, Austin, TX, USA) in accordance with the manufacturer's instructions. The RNA was treated with DNase I (AM1906; Ambion) to eliminate genomic DNA contamination and then subjected to cDNA synthesis using the miScript reverse transcription kit (218061; Qiagen, Hilden, Germany). Subsequently, real-time PCR was carried out with the miScript SYBR-Green PCR kit (218073; Qiagen) in a LightCycler (Roche Diagnostics, Mannheim, Germany) according to the manufacturer's instructions. Primers for mature miR-124 and U6 snRNA were purchased from Qiagen (MS00006622 and MS00007497, Hilden, Germany). All reactions were run in triplicate. The relative expression level of miR-124 was quantified by normalization to endogenous U6 snRNA expression level using the $2^{-\Delta \Delta \mathrm{Ct}}$ method.

Dual-luciferase reporter assay. To determine whether miR-124 can bind to the 3' untranslated region (3'UTR) of SOX9, we purchased the pEZX-SOX9 vector from GeneCopoeia (HmiT017635-MT06; Rockville, MD, USA). The pEZX-SOX9 vector contains the firefly luciferase gene with the SOX9 3'UTR and the Renilla luciferase gene. The miR-124 mimic, miR-124 mimic with mutant sequences (RiboBio, Guangzhou, China) or the miR-124 mimic control was transiently co-transfected into A549 cells with the pEZX-SOX9 vector. Cells were harvested $48 \mathrm{~h}$ after transfection and the luciferase activities were measured using the Dual-Luciferase Reporter assay system (E1910; Promega, Madison, WI, USA). Firefly luciferase activities were normalized to Renilla luciferase activities to control for transfection efficiency.

miR-124 overexpression in cultured cells. The miR-124 expression vector was constructed using BLOCK-iT ${ }^{\mathrm{TM}}$ Pol II miR RNAi expression vector kit with EmGFP (K4936-00; Invitrogen) according to the manufacturer's protocol. The negative control vector was provided by Invitrogen, which contains an insert that can form a hairpin structure just as a regular pre-miRNA, but is predicted not to target any known vertebrate gene. The expression vector or control vector was transfected into A549 cells with Lipofectamine 2000 reagent (11668-019; Invitrogen). Twenty-four hours after transfection, blasticidin (15205; Sigma, St. Louis, MO, USA) was added at a concentration of $3 \mu \mathrm{g} / \mathrm{ml}$ for 10 days. Resistant cells were analyzed by fluorescence microscopy (Nikon, Tokyo, Japan). For transient transfection, miR-124 mimics (miR10000422) or miR-124 mimic negative control (miR01201) (both from RiboBio) were transfected into A549 cells at a final concentration of $100 \mathrm{nM}$ following the provided instructions.

miR-124 knockdown. miR-124 inhibitor and miR-124 inhibitor control were purchased from Exiqon (4102198 and 199006; Vedbaek, Denmark) and transfected into A549 cells with Lipofectamine 2000 reagent, at a final concentration of $50 \mathrm{nM}$. The cells were collected $48 \mathrm{~h}$ after transfection, and the levels of miRNA-124 and SOX9 were determined by real-time RT-PCR and western blotting.

Western blotting. Total proteins in the cells and tissues were extracted using RIPA buffer (Beyotime Institute of Biotechnology, Shanghai, China). The concentration of total proteins was measured using the $\mathrm{BCA}^{\mathrm{TM}}$ protein assay kit (Pierce Biotechnology, Inc., Rockford, IL, USA). A total of $40 \mu \mathrm{g}$ proteins was separated on 12\% SDS-PAGE gels, and transferred onto nitrocellulose membranes (Millipore, Billerica, MA, USA). The membranes were blocked with $5 \%$ fat-free milk at room temperature for $2 \mathrm{~h}$, followed by incubation with the mouse anti-human primary monoclonal antibody against SOX9 (1:500; ab76997; Abcam, Cambridge, MA, USA) or GAPDH (1:5,000; D190090; Sangon Biotech, Shanghai, China) at $4^{\circ} \mathrm{C}$ overnight. The membranes were then washed in TBS for three times and incubated with horseradish peroxidase (HRP)-conjugated goat anti-mouse secondary antibody (1:5,000; D110087; Sangon Biotech) for $1 \mathrm{~h}$ at room temperature. After another three times of washing in TBS, signals from the HRP-conjugated secondary antibody were generated using enhanced chemiluminescence solution (Amersham, Piscataway, NJ, USA), and were detected by exposure of the membranes to X-ray film (Kodak, Rochester, NY, USA). The relative signal intensity was quantified by densitometry with UVIPhoto and UVISoft UVIB and application V97.04 (UVItech, Cambridge, UK).

MTT assay. After transfection with the miR-124 mimic or miR-124 inhibitor, the A549 cells were harvested, plated into 

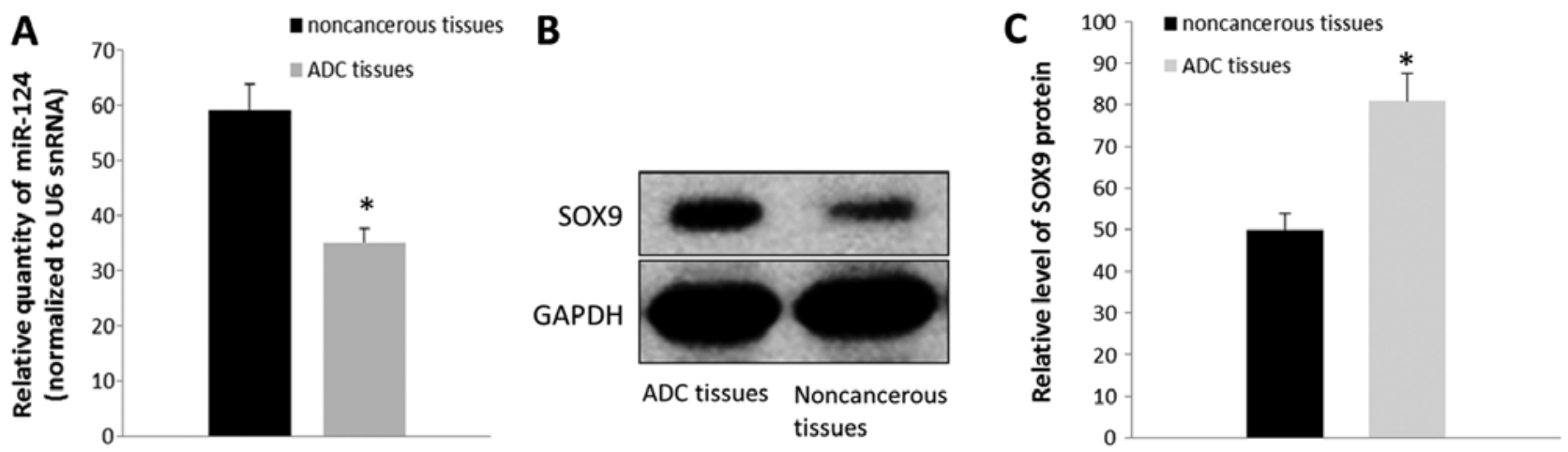

Figure 1. Expression of miR-124 and SOX9 in lung ADC tissues. (A) miR-124 expression was significantly lower in the lung ADC tissues than that in the corresponding non-cancerous tissues; ${ }^{2} \mathrm{P}<0.05$. (B) Expression of SOX9 protein in lung ADC tissues and corresponding non-cancerous tissues. SOX 9 protein levels were measured by western blot analysis and GAPDH was used as an internal control. (C) Semi-quantitative analyses of western blot analyses for SOX9 protein; ${ }^{*} \mathrm{P}<0.05$.

96-well plates $\left(2.0 \times 10^{3}\right.$ cells/well) and incubated at $37^{\circ} \mathrm{C}$. At different time points $(4,24,48$ or $72 \mathrm{~h}), 10 \mu \mathrm{l}$ of MTT reagent (5 mg/ml; Sigma-Aldrich) was added to each well and cells were incubated for another $4 \mathrm{~h}$. Then, the supernatant was discarded, and $150 \mu \mathrm{l}$ of dimethyl sulfoxide (DMSO) was added to each well. Colorimetric analysis was performed at the wavelength of $490 \mathrm{~nm}$. Data were derived from three independent experiments.

Migration and invasion assays. Cell migration and invasion capacities were measured in vitro using CytoSelect ${ }^{\mathrm{TM}}$ 24-Well Cell Migration and Invasion Assay Combo kit (Cell Biolabs, San Diego, CA, USA) according to the manufacturer's instructions. Briefly, $1 \times 10^{5}$ transfected cells were seeded into the upper chamber. For the invasion assays, the filter membrane was coated with a uniform layer of dried basement membrane matrix solution. DMEM containing $10 \%$ FBS was used as a chemoattractant, and added to the lower chamber. Following a 24-h incubation, cells on the upper side of the filters were carefully removed with cotton-tipped swabs. Invaded cells on the lower membrane were stained and colorimetric analysis was performed. The relative migratory and invasive activities were determined by the measurement of the optical density at $560 \mathrm{~nm}$ and using the value of the negative control as 1 .

Statistical analysis. All statistical analyses were performed using the SPSS 17.0 software package (SPSS, Inc., Chicago, IL, USA). The data are presented as the mean \pm standard deviation (SD). Differences between groups were analyzed using the Student's t-test. P-values are two-sided, and $\mathrm{P}<0.05$ was considered to indicate a statistically significant difference.

\section{Results}

miR-124 is downregulated in lung ADC tissues and its correlation with the SOX9 protein level. The expression levels of miR-124 in ADC tissues and corresponding adjacent non-cancerous tissues were detected by real-time RT-PCR. As shown in Fig. 1A, the results indicated that the relative level of miR-124 expression was significantly lower in the ADC specimens compared with that noted in the controls $(\mathrm{P}<0.05)$ (Fig. 1A). SOX9 protein levels were also detected by western blot analysis. The results showed that the SOX9 protein level was upregulated in the tumor samples when compared with that noted in the adjacent non-cancerous tissues $(\mathrm{P}<0.05)$ (Fig. 1B and $\mathrm{C})$. Our data suggested that the level of SOX9 protein is inversely associated with the expression of miR-124.

miR-124 suppresses the proliferative, migratory and invasive capacities of the A549 cells. To investigate the effects of miR-124 on the biological behaviors of the A549 cells, we transfected A549 cells with the miR-124 mimic or miR-124 inhibitor. Real-time RT-PCR was performed to confirm an increase or decrease in the miR-124 level $48 \mathrm{~h}$ after miR-124 mimic or miR-124 inhibitor transfection (Fig. 2A). MTT assay was utilized to evaluate cell proliferative capacity. The results showed that overexpression of miR-124 significantly suppressed cell growth of the A549 cells, whereas knockdown of miR-124 resulted in a significant increase in proliferation of the A549 cells compared with that noted in the controls (Fig. 2B and C). Transwell assay was performed to investigate the effect of miR-124 on the migration and invasion of A549 cells. As shown in Fig. 2D and E, the migratory and invasive capabilities were significantly decreased in the A549 cells transfected with the miR-124 mimic compared with these capabilities noted in the control groups. Accordingly, when A549 cells were transfected with the miR-124 inhibitor, the migratory and invasive capabilities were markedly increased compared with the controls.

SOX9 is a target gene of miR-124 in A549 cells. Using the bioinformatic tools at TargetScan database (http://www. targetcan.org/), SOX9 was identified as a candidate target of miR-124, since it contains a putative miR-124 binding site in the 3'UTR (Fig. 3A). To determine whether miR-124 can bind to its seed sequence present in the SOX9 3'UTR, we performed a luciferase reporter assay using the pEXZ-SOX9 vector containing the SOX9 3'UTR. The pEXZ-SOX9 vector was cotransfected into A549 cells with the miR-124 mimic or miR-124 mimic control. Luciferase activities were measured at $48 \mathrm{~h}$ after transfection. The results showed that overexpression of miR-124 led to a $47 \%$ decrease in luciferase expression, measured as relative luciferase activity, compared to the 


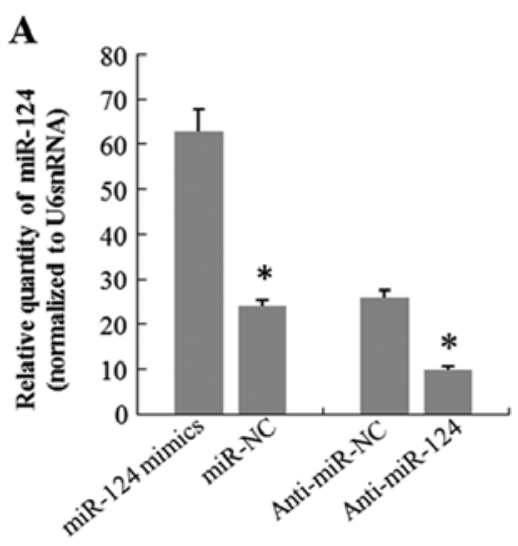

D
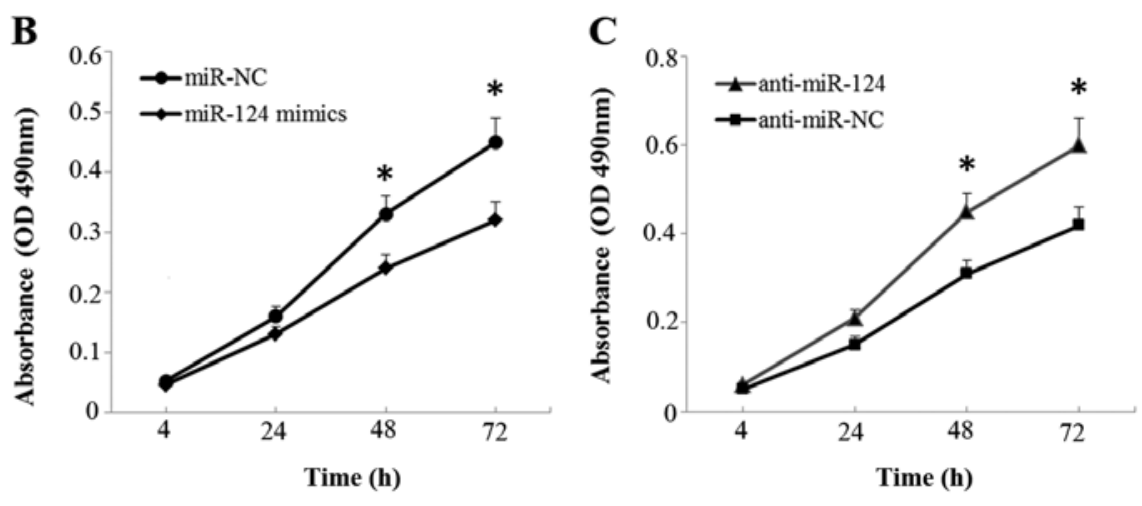

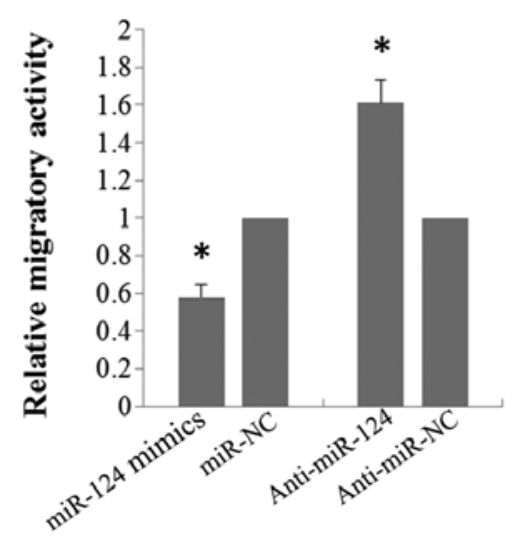

$\mathbf{E}$

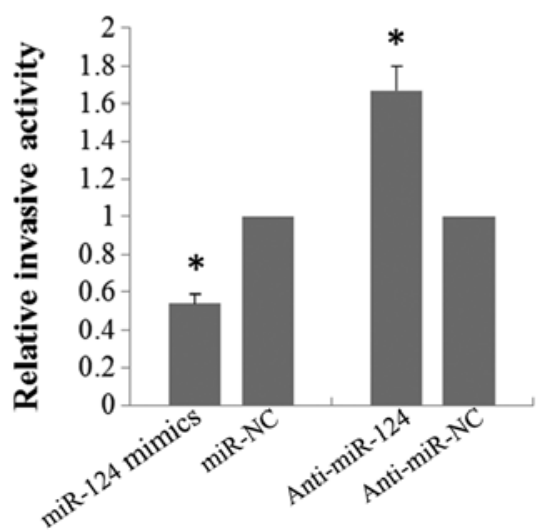

Figure 2. Effects of miR-124 on the biological behaviors of A549 cells. (A) Real-time RT-PCR analysis confirmed an increase or decrease in the miR-124 level in A549 cells transfected with the miR-124 mimics or miR-124 inhibitor (anti-miR-124); ${ }^{*}<0.05$. (B) MTT assay showed that overexpression of miR-124 reduced cell proliferation in vitro. (C) MTT assay showed that inhibition of miR-124 resulted in a significant increase in proliferation of A549 cells; ${ }^{*} \mathrm{P}<0.05$. (D) Transwell migration assay demonstrated that upregulation of miR-124 impeded the migration of A549 cells, whereas knockdown of miR-124 promoted the migration of A549 cells; * $\mathrm{P}<0.05$. (E) Transwell invasion assay showed that upregulation of miR-124 impeded the invasion of A549 cells, whereas knockdown of miR-124 promoted the invasion of A549 cells; ${ }^{*} \mathrm{P}<0.05$.

A $\begin{array}{ll}\text { SOX9 3'UTR } & \text { 5'-UUUAAGGAGGAGCUGCCUUA-3' } \\ \text { hsa-miR-124 } & \text { 3'-CCGUAAGUGGCGCACGGAAU-5' }\end{array}$

B

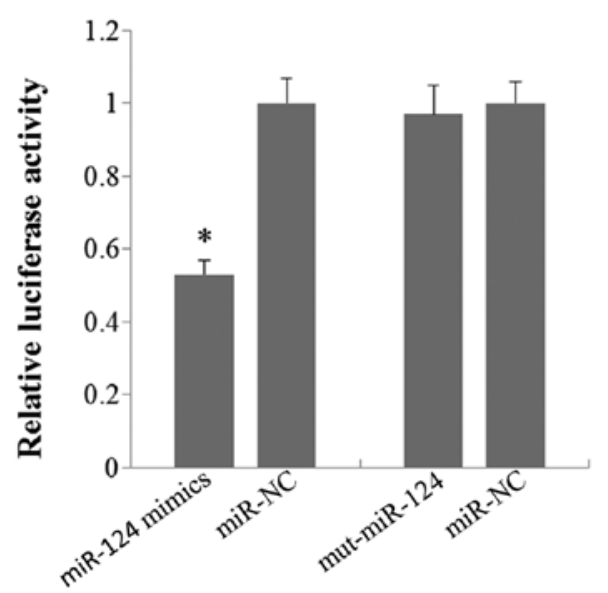

Figure 3.SOX9 is a direct target of miR-124.(A) The putative miR-124-binding sites in the 3'UTR of SOX9. (B) pEXZ-SOX9 vector was cotransfected into the A549 cells with the miR-124 mimics, mutant miR-124 or miR-124 mimic control. The relative luciferase activity was measured $48 \mathrm{~h}$ after transfection and firefly activities were normalized to Renilla luminescence; ${ }^{*} \mathrm{P}<0.05$. controls (Fig. 3B). However, when the pEXZ-SOX9 vector was cotransfected into the A549 cells with the miR-124 mimic with six mutated sequences (UCCUUACCGCGGUGAAUGCC) or the miR-124 mimic control, only a very slight effect on luciferase activity was observed (Fig. 3B). These results indicated that miR-124 was able to bind to the predicted seed sequence in the 3'UTR of SOX9 mRNA.

miR-124 regulates SOX9 protein in A549 cells. To investigate whether miR-124 affects SOX9 expression, we next examined the effect of this miRNA in a stable transfectant cell line of miR-124. Results from real-time RT-PCR showed that there was a 3.8-fold increase in miR-124 levels in the stable transfectant cell line of miR-124, as compared to cells stably transfected with the negative control vector (Fig. 4A). Western blot analysis performed on the same cells showed that the SOX9 protein level was clearly reduced (52\%) in the stable transfection cell line of miR-124 compared with the controls (Fig. 4B and C). We also knocked down miR-124 expression by transfecting A549 cells with the miR-124 inhibitor and analyzed the effects on SOX9 expression. A549 cells transfected with the miR-124 inhibitor showed a 2.4-fold decrease in the miR-124 level compared with the cells transfected with the miR-124 inhibitor control (Fig. 4A). As expected, the downregulation 

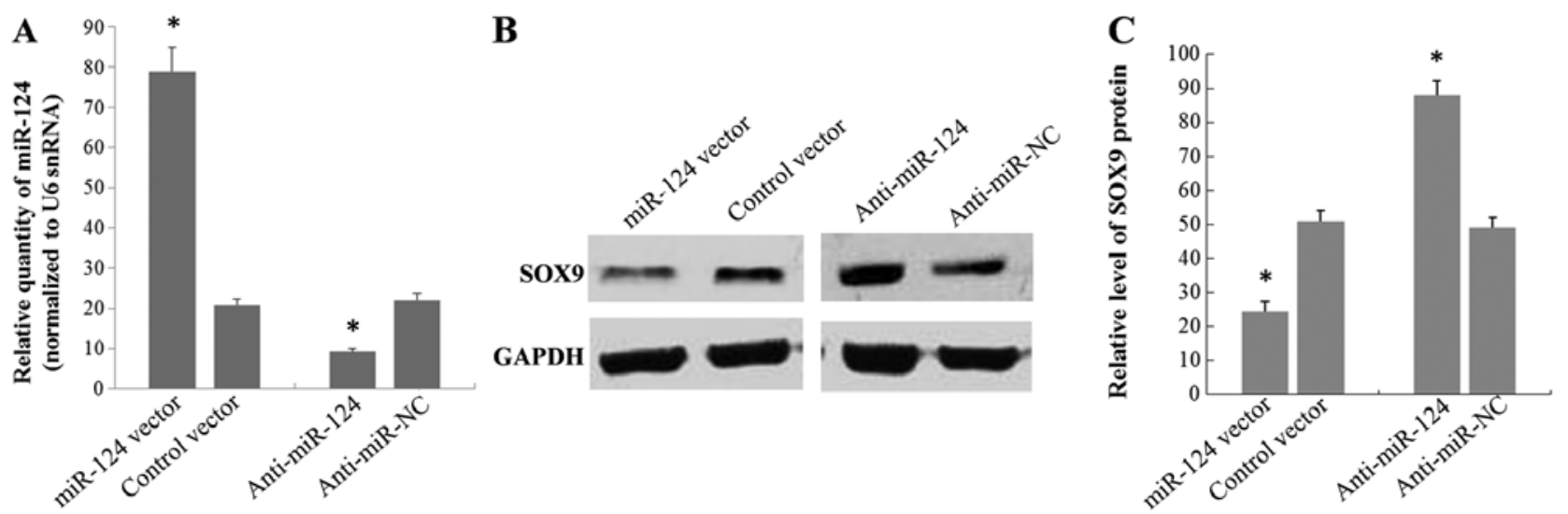

Figure 4. miR-124 regulates SOX9 protein in A549 cells. (A) Expression of miR-124 was significantly upregulated in the A549 cells transfected with the miR-124 mimics. When A549 cells were transfected with the miR-124 inhibitors, the level of miR-124 was markedly decreased; ${ }^{*} \mathrm{P}<0.05$. (B) Expression of SOX9 protein was significantly downregulated in the A549 cells transfected with the miR-124 mimics. Inhibition of miR-124 resulted in a significant increase in SOX9 protein levels in the A549 cells; ${ }^{*} \mathrm{P}<0.05$. (C) Semi-quantitative analyses of western blot analyses for SOX9 protein; ${ }^{*} \mathrm{P}<0.05$.

A
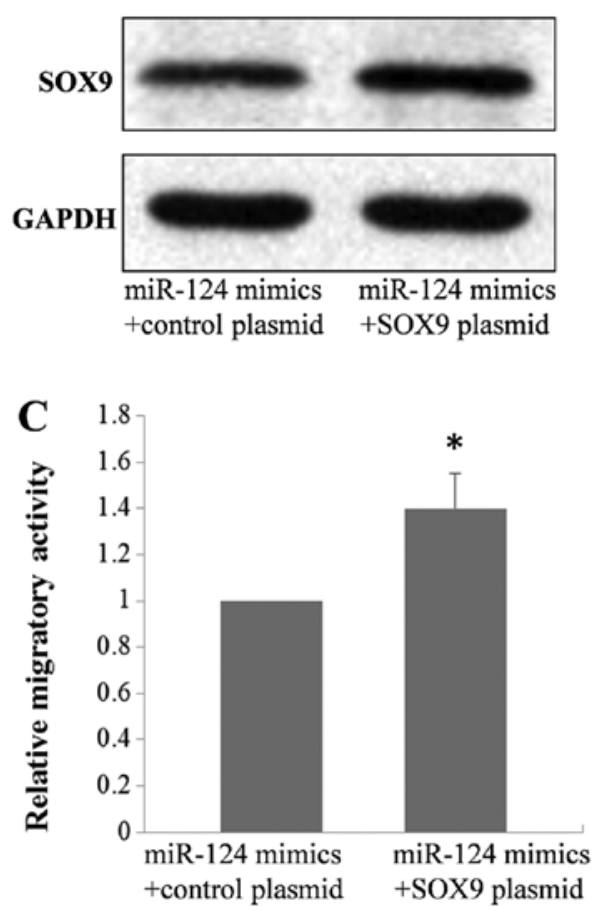
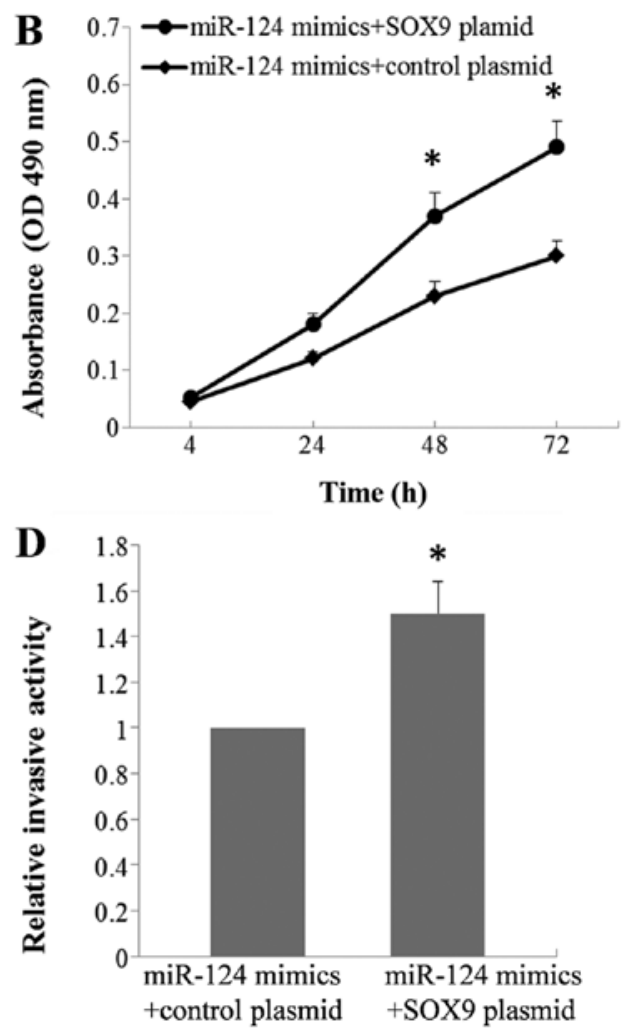

Figure 5. SOX9 is an important functional mediator of miR-124 in A549 cells. (A) miR-124 mimics were cotransfected into the A549 cells with the SOX9 expression plasmid or control plasmid. Western blot analysis showed that the level of SOX9 protein was recovered after treatment with the SOX9 plasmid. (B) MTT assay showed that recovered expression of SOX9 rescued the proliferation capacity induced by overexpression of miR-124 in the A549 cells; "P<0.05. (C) Transwell migration assay showed that exogenous expression of SOX9 rescued the migration capacity induced by overexpression of miR-124 in the A549 cells; " $\mathrm{P}<0.05$. (D) Transwell invasion assay showed that exogenous expression of SOX9 rescued the invasion capacity induced by overexpression of miR-124 in the A549 cells; ${ }^{*} \mathrm{P}<0.05$.

of miR-124 led to an increase in the SOX9 protein level of 1.8 -fold compared to the negative controls (Fig. 4B and C). Taken together, our data suggested that the expression of SOX9 is regulated by miR-124.

SOX9 is a functional mediator of miR-124 in A549 cells. In our previous study (28), we explored the functional role of SOX9 in A549 cells and showed that upregulation of SOX9 promoted cell proliferation, migration and invasion. To determine whether the suppressive effect of miR-124 on the biological behaviors of A549 cells is mediated by repression of SOX9, the miR-124 mimic was cotransfected into the A549 cells with the SOX9 plasmid or control plasmid. Western blot analysis was performed $48 \mathrm{~h}$ after transfection and the results indicated that the protein level of SOX9 was recovered after treatment with the SOX9 plasmid compared to the controls (Fig. 5A). 
Moreover, MTT, migration and invasion assays showed that exogenous expression of SOX9 was able to restore the proliferative, migratory and invasive activity compared with these cell behaviors noted in the controls (Fig. 5B-D). This indicated that the suppressive effect of miR-124 was mediated by repression of SOX9, and SOX9 is a functional mediator of miR-124 in A549 cells.

\section{Discussion}

The dysregulation of miRNAs is involved in the initiation and progression of various types of tumors. However, their potential roles in lung adenocarcinoma (ADC) remain unclear. In the present study, we found that miR-124 was downregulated in ADC tissues and the level of miR-124 was inversely correlated with SOX9 expression. Moreover, the functional assays in human lung ADC cell line A549 showed that enforced expression of miR-124 significantly inhibited cell proliferation, migration and invasion. When A549 cells were transfected with the miR-124 expression vector or miR-124 inhibitor, the SOX9 protein level was downregulated or upregulated, which indicated that miR-124 could regulate SOX9 expression. The luciferase reporter assay confirmed that SOX9 is a direct target of miR-124. Finally, we showed that exogenous expression of SOX9 rescued the phenotype induced by overexpression of miR-124 in the A549 cells. These results suggested that miR-494 affects cell proliferation, migration and invasion by directly regulating SOX9 expression in lung ADC.

Previous studies have shown that miR-124 is associated with several human malignancies and it is a putative tumor suppressor. However, the function of miR-124 in lung ADC remains unclear. In the present study, our results showed that miR-124 was downregulated in the tissues of lung ADC patients using real-time RT-PCR assays, and we focused on the functions of miR-124 in the proliferation, migration and invasion of lung ADC cells. Using human lung ADC cell line A549 transfected with the miR-124 mimic or miR-124 inhibitor, we demonstrated that miR-124 was functionally involved in suppression of cell proliferation, migration and invasion.

SOX9, which belongs to a family of genes called SOX [SRY (sex determining region Y)-boxes], is a key regulator of embryonic development. Emerging evidence has provided a link between SOX9 and cancer development. In some types of cancers, SOX9 is upregulated and functions as an oncogene, whereas in other types of cancers, SOX9 is downregulated and functions as a tumor suppressor. Our previous study found that SOX9 was upregulated in lung ADC and promoted cell proliferation, migration and invasion. In the present study, we found that SOX9 is a direct target gene of miR-124. More importantly, exogenous expression of SOX9 restored the proliferative, migratory and invasive activities induced by overexpression of miR-124 in A549 cells. All these results suggest that miR-124 can inhibit cell proliferation, migration and invasion by directly targeting SOX9 and SOX9 is an important functional mediator of miR-124 in lung ADC cells. As we know, there exists a complicated connection between miRNAs and their target genes. One miRNA can have many targets and several miRNAs can have the same target. Some genes, such as talin 1, PDE4B and STAT3, have also been found to be the target of miR-124 in several human malig- nancies $(4,15,29)$. SOX9 may not be the only miR-124 target dysregulated in lung ADC. Thus, further studies are needed to investigate the potential functional targets of miR-124, and the actual mechanisms by which miR-124 affects lung ADC progression require further clarification.

In conclusion, the present study demonstrated that miR-124 was downregulated in tissues of lung ADC patients. Moreover, miR-124 inhibited cell proliferation, migration and invasion of A549 cells. Finally, we confirmed that miR-124 directly targets SOX9 by binding to the 3'UTR of SOX9 and SOX9 was found to be an important functional mediator of miR-124 in A549 cells. Overall, our findings indicate that miR-124 functions as a tumor suppressor in lung ADC and may be a promising candidate for miR-based therapy against lung ADC.

\section{Acknowledgements}

The present study was supported by grants from the Shandong Provincial Natural Science Foundation of China (grant no. ZR2015PH038), and the Seed Fund of the Second Hospital of Shandong University (grant no. S2014010018).

\section{References}

1. Wang Y, Huang C, Chintagari NR, Xi D, Weng T and Liu L: miR-124 regulates fetal pulmonary epithelial cell maturation. Am J Physiol Lung Cell Mol Physiol 309: L400-L413, 2015.

2. Osada $\mathrm{H}$ and Takahashi T: MicroRNAs in biological processes and carcinogenesis. Carcinogenesis 28: 2-12, 2007.

3. Lovat F, Valeri N and Croce CM: MicroRNAs in the pathogenesis of cancer. Semin Oncol 38: 724-733, 2011.

4. Zhang W, Mao YQ, Wang H, Yin WJ, Zhu SX and Wang WC: MiR-124 suppresses cell motility and adhesion by targeting talin 1 in prostate cancer cells. Cancer Cell Int 15: 49, 2015.

5. Dong LL, Chen LM, Wang WM and Zhang LM: Decreased expression of microRNA-124 is an independent unfavorable prognostic factor for patients with breast cancer. Diagn Pathol 10: $45,2015$.

6. Xi ZW, Xin SY, Zhou LQ, Yuan HX, Wang Q and Chen KX: Downregulation of rho-associated protein kinase 1 by miR-124 in colorectal cancer. World J Gastroenterol 21: 5454-5464, 2015.

7. Wan HY, Li QQ, Zhang Y, Tian W, Li YN, Liu M, Li X and Tang H: MiR-124 represses vasculogenic mimicry and cell motility by targeting amotL1 in cervical cancer cells. Cancer Lett 355: 148-158, 2014.

8. Hu CB, Li QL, Hu JF, Zhang Q, Xie JP and Deng L: miR-124 inhibits growth and invasion of gastric cancer by targeting ROCK1. Asian Pac J Cancer Prev 15: 6543-6546, 2014.

9. Peng XH, Huang HR, Lu J, Liu X, Zhao FP, Zhang B, Lin SX, Wang L, Chen HH, Xu X, et al: MiR-124 suppresses tumor growth and metastasis by targeting Foxq1 in nasopharyngeal carcinoma. Mol Cancer 13: 186, 2014.

10. Zhang T, Wang J, Zhai X, Li H, Li C and Chang J: MiR-124 retards bladder cancer growth by directly targeting CDK4. Acta Biochim Biophys Sin 46: 1072-1079, 2014.

11. Zhang H, Wang Q, Zhao Q and Di W: MiR-124 inhibits the migration and invasion of ovarian cancer cells by targeting SphK1. J Ovarian Res 6: 84, 2013.

12. Lu Y, Yue X, Cui Y, Zhang J and Wang K: MicroRNA-124 suppresses growth of human hepatocellular carcinoma by targeting STAT3. Biochem Biophys Res Commun 441: 873-879, 2013.

13. Zhang Y, Li H, Han J and Zhang Y: Down-regulation of microRNA-124 is correlated with tumor metastasis and poor prognosis in patients with lung cancer. Int J Clin Exp Pathol 8: 1967-1972, 2015

14. Sun Y, Ai X, Shen S and Lu S: NF-кB-mediated miR-124 suppresses metastasis of non-small-cell lung cancer by targeting MYO10. Oncotarget 6: 8244-8254, 2015. 
15. Li X, Yu Z, Li Y, Liu S, Gao C, Hou X, Yao R and Cui L: The tumor suppressor miR-124 inhibits cell proliferation by targeting STAT3 and functions as a prognostic marker for postoperative NSCLC patients. Int J Oncol 46: 798-808, 2015.

16. Chaboissier MC, Kobayashi A, Vidal VI, Lützkendorf S van de Kant HJ, Wegner M, de Rooij DG, Behringer RR and Schedl A: Functional analysis of Sox 8 and Sox 9 during sex determination in the mouse. Development 131: 1891-1901, 2004.

17. Akiyama H, Chaboissier MC, Martin JF, Schedl A and de Crombrugghe B: The transcription factor Sox 9 has essential roles in successive steps of the chondrocyte differentiation pathway and is required for expression of Sox5 and Sox6. Genes Dev 16: 2813-2828, 2002.

18. Bruun J, Kolberg M, Nesland JM, Svindland A, Nesbakken A and Lothe RA: Prognostic significance of $\beta$-catenin, E-cadherin, and SOX9 in colorectal cancer: Results from a large populationrepresentative series. Front Oncol 4: 118, 2014.

19. Jiang SS, Fang WT, Hou YH, Huang SF, Yen BL, Chang JL, Li SM, Liu HP, Liu YL, Huang CT, et al: Upregulation of SOX9 in lung adenocarcinoma and its involvement in the regulation of cell growth and tumorigenicity. Clin Cancer Res 16: 4363-4373, 2010.

20. Qin GQ, He HC, Han ZD, Liang YX, Yang SB, Huang YQ, Zhou L, Fu H, Li JX, Jiang FN, et al: Combined overexpression of HIVEP3 and SOX9 predicts unfavorable biochemical recurrence-free survival in patients with prostate cancer. Onco Targets Ther 7: 137-146, 2014.

21. Müller P, Crofts JD, Newman BS, Bridgewater LC, Lin CY, Gustafsson JA and Ström A: SOX9 mediates the retinoic acidinduced HES-1 gene expression in human breast cancer cells. Breast Cancer Res Treat 120: 317-326, 2010.
22. Xia S, Feng Z, Qi X, Yin Y, Jin J, Wu Y, Wu H, Feng Y and Tao M: Clinical implication of Sox9 and activated Akt expression in pancreatic ductal adenocarcinoma. Med Oncol 32: 358, 2015.

23. Wang HY, Lian P and Zheng PS: SOX9, a potential tumor suppressor in cervical cancer, transactivates $\mathrm{p} 21^{\mathrm{WAF} 1 / \mathrm{CIP} 1}$ and suppresses cervical tumor growth. Oncotarget 6: 20711-20722, 2015.

24. Zhu D, Chen H, Yang X, Chen W, Wang L, Xu J and Yu L: miR-32 functions as a tumor suppressor and directly targets SOX9 in human non-small cell lung cancer. Onco Targets Ther 8: 1773-1783, 2015.

25. Li X, Wang ZX, Wang ZS and Li QF: Effect of microRNA-101 on apoptosis of rabbit condylar cartilage cells by inhibiting target gene SOX9. Asian Pac J Trop Med 8: 502-505, 2015.

26. Mak IW, Singh S, Turcotte R and Ghert M: The epigenetic regulation of SOX9 by miR-145 in human chondrosarcoma. J Cell Biochem 116: 37-44, 2015.

27. Real FM, Sekido R, Lupiáñez DG, Lovell-Badge R, Jiménez R and Burgos M: A microRNA (mmu-miR-124) prevents Sox 9 expression in developing mouse ovarian cells. Biol Reprod 89: 78, 2013.

28. Wang X, Ju Y, Zhou MI, Liu X and Zhou C: Upregulation of SOX 9 promotes cell proliferation, migration and invasion in lung adenocarcinoma. Oncol Lett 10: 990-994, 2015.

29. Kim J, Jeong D, Nam J, Aung TN, Gim JA, Park KU and Kim SW: MicroRNA-124 regulates glucocorticoid sensitivity by targeting phosphodiesterase 4B in diffuse large B cell lymphoma. Gene 558: 173-180, 2015. 